\title{
NEW STABILITY TESTS OF POSITIVE 1D AND 2D LINEAR SYSTEMS
}

\author{
Tadeusz Kaczorek \\ Bialystok University of Technology \\ Faculty of Electrical Engineering \\ Wiejska 45D, 15-351 Bialystok \\ e-mail: kaczorek@isep.pw.edu.pl
}

\section{KEYWORDS}

positive, linear, 1D, 2D system, asymptotic stability, test.

\begin{abstract}
New tests for checking asymptotic stability of positive 1D continuous-time and discrete-time linear systems without and with delays and of positive 2D linear systems described by the general and the Roesser models are proposed. Checking of the asymptotic stability of positive 2D linear systems is reduced to checking of suitable corresponding 1D positive linear systems. Effectiveness of the tests is shown on numerical examples.
\end{abstract}

\section{INTRODUCTION}

A dynamical system is called positive if its trajectory starting from any nonnegative initial state remains forever in the positive orthant for all nonnegative inputs. An overview of state of the art in positive theory is given in the monographs (Farina and Rinaldi 2000; Kaczorek 2002). Variety of models having positive behavior can be found in engineering, economics, social sciences, biology and medicine, etc..

New stability conditions for discrete-time linear systems have been proposed in (Busłowicz 2008) and next have been extended to robust stability of fractional discretetime linear systems in (Busłowicz 2010). The stability of positive continuous-time linear systems with delays has been addressed in (Kaczorek 2009c) The independence of the asymptotic stability of positive 2D linear systems with delays of the number and values of the delays has been shown in (Kaczorek 2009d). The asymptotic stability of positive 2D linear systems without and with delays has been considered in (Kaczorek 2009a and $2009 \mathrm{~b})$. The stability and stabilization of positive fractional linear systems by state-feedbacks have been analyzed in (Kaczorek 2010).

In this paper new tests for checking asymptotic stability of positive 1D continuous-time and discrete-time linear systems without and with delays and of positive 2D linear systems described by the general and the Roesser models will be proposed. It will be shown that the checking of the asymptotic stability of positive 2D linear systems can be reduced to checking of stability of suitable corresponding 1D positive linear systems.
The paper is organized as follows. In section 2 new stability tests for positive continuous-time linear systems are proposed. An extension of these tests for positive discrete-time linear systems is given in section 3 . Application of the tests to checking the asymptotic stability of positive 1D linear systems with delays is given in section 4 . In section 5 the tests are applied to positive 2D linear systems described by the general and Roesser models. Concluding remarks are given in section 6 .

The following notation will be used: $\mathfrak{R}$ - the set of real numbers, $\Re^{n \times m}$ - the set of $n \times m$ real matrices, $\Re_{+}^{n \times m}$ the set of $n \times m$ matrices with nonnegative entries and $\mathfrak{R}_{+}^{n}=\mathfrak{R}_{+}^{n \times 1}, M_{n}$ - the set of $n \times n$ Metzler matrices (real matrices with nonnegative off-diagonal entries), $I_{n}$ - the $n \times n$ identity matrix.

\section{CONTINUOUS-TIME LINEAR SYSTEMS}

Consider the continuous-time linear system

$$
\dot{x}(t)=A x(t)
$$

where $x(t) \in \mathfrak{R}^{n}$ is the state vector and $A \in \mathfrak{R}^{n \times n}$.

The system (2.1) is called (internally) positive if $x(t) \in \mathfrak{R}_{+}^{n}, \quad t \geq 0 \quad$ for any initial conditions $x(0)=x_{0} \in \mathfrak{R}_{+}^{n} \quad$ (Farina and Rinaldi 2000; Kaczorek 2002).

Theorem 2.1. (Farina and Rinaldi 2000; Kaczorek 2002) The system (2.1) is positive if and only if $A$ is a Metzler matrix.

The positive system is called asymptotically stable if

$$
\lim _{t \rightarrow \infty} x(t)=\lim _{t \rightarrow \infty} e^{A t} x_{0}=0 \text { for all } x_{0} \in \Re_{+}^{n}
$$

Theorem 2.2. (Farina and Rinaldi 2000; Kaczorek 2002) The positive system (2.1) is asymptotically stable if and only if all principal minors $M_{i}, i=1, \ldots, n$ of the matrix $-A$ are positive, i.e.

$$
\begin{aligned}
& M_{1}=-a_{11}>0, \quad M_{2}=\left|\begin{array}{ll}
-a_{11} & -a_{12} \\
-a_{21} & -a_{22}
\end{array}\right|>0, \ldots, \\
& M_{n}=\operatorname{det}[-A]>0
\end{aligned}
$$


Theorem 2.3. (Farina and Rinaldi 2000; Kaczorek 2002) The positive system (2.1) is asymptotically stable only if all diagonal entries of the matrix $A$ are negative.

Let $A=\left[a_{i j}\right] \in \mathfrak{R}^{n \times n}$ be a Metzler matrix with negative diagonal entries $\left(a_{i i}<0, \quad i=1, \ldots, n\right)$.

Let define

$$
\begin{aligned}
& A_{n}^{(0)}=A=\left[\begin{array}{ccc}
a_{11}^{(0)} & \ldots & a_{1, n}^{(0)} \\
\vdots & \ldots & \vdots \\
a_{n, 1}^{(0)} & \ldots & a_{n, n}^{(0)}
\end{array}\right]=\left[\begin{array}{cc}
A_{n-1}^{(0)} & b_{n-1}^{(0)} \\
c_{n-1}^{(0)} & a_{n, n}^{(0)}
\end{array}\right], \\
& A_{n-1}^{(0)}=\left[\begin{array}{ccc}
a_{11}^{(0)} & \ldots & a_{1, n-1}^{(0)} \\
\vdots & \ldots & \vdots \\
a_{n-1,1}^{(0)} & \ldots & a_{n-1, n-1}^{(0)}
\end{array}\right] \\
& b_{n-1}^{(0)}=\left[\begin{array}{c}
a_{1, n}^{(0)} \\
\vdots \\
a_{n-1, n}^{(0)}
\end{array}\right], \quad c_{n-1}^{(0)}=\left[\begin{array}{lll}
a_{n, 1}^{(0)} & \ldots & a_{n, n-1}^{(0)}
\end{array}\right]
\end{aligned}
$$

and

$$
\begin{aligned}
A_{n-k}^{(k)} & =A_{n-k}^{(k-1)}-\frac{b_{n-k}^{(k-1)} c_{n-k}^{(k-1)}}{a_{n-k+1, n-k+1}^{(k-1)}} \\
& =\left[\begin{array}{ccc}
a_{11}^{(k)} & \ldots & a_{1, n-k}^{(k)} \\
\vdots & \ldots & \vdots \\
a_{n-k, 1}^{(k)} & \ldots & a_{n-k, n-k}^{(k)}
\end{array}\right]=\left[\begin{array}{cc}
A_{n-k-1}^{(k)} & b_{n-k-1}^{(k)} \\
c_{n-k-1}^{(k)} & a_{n-k, n-k}^{(k)}
\end{array}\right], \\
b_{n-k-1}^{(k)} & =\left[\begin{array}{c}
a_{1, n-k}^{(k)} \\
\vdots \\
a_{n-k-1, n-k}^{(k)}
\end{array}\right], c_{n-k-1}^{(k)}=\left[\begin{array}{lll}
a_{n-k, 1}^{(k)} & \ldots & a_{n-k, n-k-1}^{(k)}
\end{array}\right]
\end{aligned}
$$

for $k=1, \ldots, n-1$.

Let us denote by $L[i+j \times c]$ the following elementary row operation on the matrix $A$ : addition to the $i$-th row the $j$-th row multiplied by a scalar $c$. It is well-known that using these elementary operation we may reduced the matrix

$$
A=\left[\begin{array}{cccc}
a_{11} & a_{12} & \ldots & a_{1, n} \\
a_{21} & a_{22} & \ldots & a_{2, n} \\
\vdots & \vdots & \ldots & \vdots \\
a_{n, 1} & a_{n, 2} & \ldots & a_{n, n}
\end{array}\right]
$$

to the lower triangular form

$$
\tilde{A}=\left[\begin{array}{cccc}
\tilde{a}_{11} & 0 & \ldots & 0 \\
\tilde{a}_{21} & \tilde{a}_{22} & \ldots & 0 \\
\vdots & \vdots & \ddots & \vdots \\
\tilde{a}_{n, 1} & \tilde{a}_{n, 2} & \ldots & \tilde{a}_{n, n}
\end{array}\right]
$$

It is easy to show that if the matrix (2.4) is Metzler matrix with negative diagonal entries then the matrix (2.5) is also a Metzler matrix.

Theorem 2.4. The positive system with the matrix (2.5) is asymptotically stable if and only if all diagonal entries of the matrix are negative.

Proof. The eigenvalues of the matrix (2.5) are equal to its diagonal entries $\tilde{a}_{11}, \ldots, \tilde{a}_{n n}$ and the positive system is asymptotically stable if and only if all the diagonal entries are negative.

Theorem 2.5. The positive continuous-time linear system (2.1) is asymptotically stable if and only if one of the equivalent conditions is satisfied:

i) the diagonal entries of the matrices defined by (2.3)

$$
A_{n-k}^{(k)} \text { for } k=1, \ldots, n-1
$$

are negative,

ii) the diagonal entries of the lower triangular matrix (2.5) are negative, i.e.

$$
\tilde{a}_{k k}<0 \text { for } k=1, \ldots, n
$$

Proof. To simplify the notation we shall show the equivalency of the conditions (2.2) and (2.6) for $n=3$. From Theorem 2.2 for $n=3$ we have

$$
\begin{aligned}
& -M_{1}=a_{11}<0, \quad(-1)^{2} M_{2}=a_{11} a_{22}-a_{12} a_{21}>0, \\
& (-1)^{3} M_{3}=\operatorname{det}\left[\begin{array}{lll}
a_{11} & a_{12} & a_{13} \\
a_{21} & a_{22} & a_{23} \\
a_{31} & a_{32} & a_{33}
\end{array}\right] \\
& =a_{33} \operatorname{det}\left\{\left[\begin{array}{ll}
a_{11} & a_{12} \\
a_{21} & a_{22}
\end{array}\right]-\frac{1}{a_{33}}\left[\begin{array}{l}
a_{13} \\
a_{23}
\end{array}\right]\left[\begin{array}{ll}
a_{31} & a_{32}
\end{array}\right]\right\} \\
& =a_{33} \operatorname{det}\left\{\frac{1}{a_{33}}\left[\begin{array}{ll}
a_{11} a_{33}-a_{13} a_{31} & a_{12} a_{33}-a_{13} a_{32} \\
a_{21} a_{33}-a_{23} a_{31} & a_{22} a_{33}-a_{23} a_{32}
\end{array}\right]\right\}<0 .
\end{aligned}
$$

By condition i) of Theorem 2.5 for $n=3$ the diagonal entries of the matrices

$$
\begin{aligned}
& A_{2}^{(1)}=A_{2}^{(0)}-\frac{b_{2}^{(0)} c_{2}^{(0)}}{a_{33}^{(0)}}=\left[\begin{array}{ll}
a_{11} & a_{12} \\
a_{21} & a_{22}
\end{array}\right]-\frac{1}{a_{33}}\left[\begin{array}{l}
a_{13} \\
a_{23}
\end{array}\right]\left[\begin{array}{ll}
a_{31} & a_{32}
\end{array}\right] \\
& =\frac{1}{a_{33}}\left[\begin{array}{ll}
a_{11} a_{33}-a_{13} a_{31} & a_{12} a_{33}-a_{13} a_{32} \\
a_{21} a_{33}-a_{23} a_{31} & a_{22} a_{33}-a_{23} a_{32}
\end{array}\right]=\left[\begin{array}{ll}
\bar{a}_{11} & \bar{a}_{12} \\
\bar{a}_{21} & \bar{a}_{22}
\end{array}\right] \\
& A_{1}^{(2)}=A_{1}^{(1)}-\frac{b_{1}^{(1)} c_{1}^{(1)}}{a_{22}^{(1)}}=\bar{a}_{11}-\frac{\bar{a}_{12} \bar{a}_{21}}{\bar{a}_{22}}=\frac{\bar{a}_{11} \bar{a}_{22}-\bar{a}_{12} \bar{a}_{21}}{\bar{a}_{22}}
\end{aligned}
$$

are negative. Note that the condition (2.8) are equivalent to the conditions (2.9) since $a_{i i}<0, \quad i=1,2,3$ and the inequalities

$$
\bar{a}_{11}=\frac{a_{11} a_{33}-a_{13} a_{31}}{a_{33}}<0, \bar{a}_{22}=\frac{a_{22} a_{33}-a_{23} a_{32}}{a_{33}}<0
$$


and

$$
\frac{\bar{a}_{11} \bar{a}_{22}-\bar{a}_{12} \bar{a}_{21}}{\bar{a}_{22}}<0
$$

are satisfied if and only if $\operatorname{det}\left[\begin{array}{ll}a_{11} & a_{12} \\ a_{21} & a_{22}\end{array}\right]>0$ and $\operatorname{det}\left\{\frac{1}{a_{33}}\left[\begin{array}{ll}a_{11} a_{33}-a_{13} a_{31} & a_{12} a_{33}-a_{13} a_{32} \\ a_{21} a_{33}-a_{23} a_{31} & a_{22} a_{33}-a_{23} a_{32}\end{array}\right]\right\}>0$.

The proof can be also accomplished by induction with respect to $n$. A different proof is given in (Narendra and Shorten 2010).

To show the equivalence of the conditions (2.6) and (2.7) note that the computation of the matrix $A_{n-1}^{(1)}$ by the use of (2.3b) for $k=1$ is equivalent to the reduction to zero of the entries $a_{j, n}, j=1, \ldots, n-1$ of the matrix (2.4) by elementary row operations since

$$
A_{n-1}^{(1)}=\left[\begin{array}{ccc}
a_{11} & \ldots & a_{1, n-1} \\
\vdots & \ldots & \vdots \\
a_{n-1,1} & \ldots & a_{n-1, n-1}
\end{array}\right]-\frac{1}{a_{n, n}}\left[\begin{array}{c}
a_{1, n} \\
\vdots \\
a_{n-1, n}
\end{array}\right]\left[\begin{array}{lll}
a_{n, 1} & \ldots & a_{n, n-1}
\end{array}\right]
$$

Note that $-\frac{a_{i, n}}{a_{n, n}}>0$ for $i=1, \ldots, n-1$ and $-\frac{a_{n, i} a_{1 n}}{a_{n, n}}>0$ for $i=1, \ldots, n-1$ since $a_{n, n}<0$ and $a_{i, j} \geq 0$ for $i \neq j$. Thus, the matrix $A_{n-1}^{(1)}$ is a Metzler matrix. Continuing this procedure after $n$ steps we obtain the Metzler lower triangular matrix (2.5). Therefore, the conditions (2.6) and (2.7) are equivalent. $\square$

Example 2.1. Consider the positive system (2.1) with the matrix

$$
A=\left[\begin{array}{ccc}
-2 & 1 & 0 \\
0 & -1 & 1 \\
1 & 1 & -2
\end{array}\right]
$$

Check the asymptotic stability using the conditions (2.2), (2.6) and (2.7).

Using (2.2) for (2.11) we obtain

$$
\begin{aligned}
& M_{1}=2>0, \quad M_{2}=\left|\begin{array}{cc}
2 & -1 \\
0 & 1
\end{array}\right|=2>0, \\
& M_{3}=\operatorname{det}[-A]=\left|\begin{array}{ccc}
2 & -1 & 0 \\
0 & 1 & -1 \\
-1 & -1 & 2
\end{array}\right|=1>0
\end{aligned}
$$

The conditions (2.2) of Theorem 2.2 are satisfied and the positive system (2.1) with (2.11) is asymptotically stable.

Using (2.3) for (2.11) we obtain

$$
\begin{aligned}
A_{2}^{(1)} & =A_{2}^{(0)}-\frac{b_{2}^{(0)} c_{2}^{(0)}}{a_{33}^{(0)}} \\
& =\left[\begin{array}{cc}
-2 & 1 \\
0 & -1
\end{array}\right]+\frac{1}{2}\left[\begin{array}{l}
0 \\
1
\end{array}\right]\left[\begin{array}{ll}
1 & 1
\end{array}\right]=\left[\begin{array}{cc}
-2 & 1 \\
0.5 & -0.5
\end{array}\right] \\
A_{1}^{(2)} & =A_{1}^{(1)}-\frac{b_{1}^{(1)} c_{1}^{(1)}}{a_{22}^{(1)}}=-2+\frac{0.5}{0.5}=-1
\end{aligned}
$$

The conditions (2.6) of Theorem 2.5 are satisfied and the positive system is asymptotically stable.

Using the elementary row operations to the matrix (2.11) we obtain

$$
\begin{aligned}
A= & {\left[\begin{array}{ccc}
-2 & 1 & 0 \\
0 & -1 & 1 \\
1 & 1 & -2
\end{array}\right] \stackrel{L[2+3 \times 0.5]}{\longrightarrow}\left[\begin{array}{ccc}
-2 & 1 & 0 \\
0.5 & -0.5 & 0 \\
1 & 1 & -2
\end{array}\right] } \\
& \stackrel{L[1+2 \times 2]}{\longrightarrow}\left[\begin{array}{ccc}
-1 & 0 & 0 \\
0.5 & -0.5 & 0 \\
1 & 1 & -2
\end{array}\right] .
\end{aligned}
$$

The conditions (2.7) of Theorem 2.5 are also satisfied and the positive system is asymptotically stable.

\section{DISCRETE-TIME LINEAR SYSTEMS}

Consider the discrete-time linear system

$$
x_{i+1}=\bar{A} x_{i}, \quad i \in Z_{+}=\{0,1, \ldots\}
$$

where $x_{i} \in \mathfrak{R}^{n}$ is the state vector and $\bar{A} \in \mathfrak{R}^{n \times n}$.

The system (3.1) is called (internally) positive if $x_{i} \in \mathfrak{R}_{+}^{n}, i \in Z_{+}$for any initial conditions $x_{0} \in \mathfrak{R}_{+}^{n}$.

Theorem 3.1. (Farina and Rinaldi 2000; Kaczorek 2002) The system (3.1) is positive if and only if $A \in \mathfrak{R}_{+}^{n \times n}$.

The positive system is called asymptotically stable if

$$
\lim _{i \rightarrow \infty} x_{i}=\lim _{i \rightarrow \infty} A^{i} x_{0}=0 \text { for all } x_{0} \in \Re_{+}^{n}
$$

From Theorem 2.2 and 3.1 it follows that the nonnegative matrix $\bar{A}$ is asymptotically stable if and only if the Metzler matrix $\bar{A}-I_{n}$ is asymptotically sable.

Theorem 3.2. (Farina and Rinaldi 2000; Kaczorek 2002) The positive system (3.1) is asymptotically stable if and only if all principal minors $\hat{M}_{i}, \quad i=1, \ldots, n$ of the matrix $\hat{A}=I_{n}-\bar{A}=\left[\hat{a}_{i j}\right] \in \mathfrak{R}^{n \times n}$ are positive, i.e.

$$
\hat{M}_{1}=\hat{a}_{11}>0, \quad \hat{M}_{2}=\left|\begin{array}{ll}
\hat{a}_{11} & \hat{a}_{12} \\
\hat{a}_{21} & \hat{a}_{22}
\end{array}\right|>0, \ldots, \quad \hat{M}_{n}=\operatorname{det}[\hat{A}]>0
$$


Theorem 3.3. (Farina and Rinaldi 2000; Kaczorek 2002) The positive system (3.1) is asymptotically stable only if all diagonal entries of the matrix $\bar{A}$ are less than 1 . It is assumed that $\bar{a}_{i i}<1, i=1, \ldots, n$ of the matrix $\bar{A}=\left[\bar{a}_{i j}\right] \in \mathfrak{R}_{+}^{n \times n}$ since otherwise by Theorem 3.3 the system is unstable. Using (2.3) in a similar way as for the matrix $A$ we define for the matrix $\hat{A}=\bar{A}-I_{n}=\left[\hat{a}_{i j}\right]$ the matrices $\hat{A}_{n-k}^{(k)}$ for $k=0,1, \ldots, n-1$. Using the elementary row operations we reduce the matrix $\hat{A}$ to the lower triangular form

$$
\tilde{A}^{\prime}=\left[\begin{array}{cccc}
\tilde{a}_{11}^{\prime} & 0 & \ldots & 0 \\
\tilde{a}_{21}^{\prime} & \tilde{a}_{22}^{\prime} & \ldots & 0 \\
\vdots & \vdots & \ddots & \vdots \\
\tilde{a}_{n, 1}^{\prime} & \tilde{a}_{n, 2}^{\prime} & \ldots & \tilde{a}_{n, n}^{\prime}
\end{array}\right]
$$

Theorem 3.4. The positive discrete-time system with the matrix (3.4) is asymptotically stable if and only if all diagonal entries of the matrix $\hat{A}^{\prime}$ are less than 1 .

Proof is similar to the proof of Theorem 2.4.

Theorem 3.5. The positive discrete-time linear system (3.1) is asymptotically stable if and only if one of the equivalent conditions is satisfied:

i) the diagonal entries of the matrices

$$
\hat{A}_{n-k}^{(k)} \text { for } k=1, \ldots, n-1
$$

are negative,

ii) the diagonal entries of the lower triangular matrix (3.4) are negative, i.e.

$$
\hat{a}_{k k}^{\prime}<0 \text { for } k=1, \ldots, n
$$

Proof. The positive discrete-time system (3.1) is asymptotically stable if and only if the corresponding continuous-time system with the Metzler matrix $\hat{A}=\bar{A}-I_{n}$ is asymptotically stable. By Theorem 2.5 the positive discrete-time system (3.1) is asymptotically stable if one of its conditions is satisfied.

Example 3.1. Check the asymptotic stability of the positive system (3.1) with the matrix

$$
\bar{A}=\left[\begin{array}{ll}
0.5 & 0.1 \\
0.2 & 0.4
\end{array}\right]
$$

In this case

$$
\hat{A}=\bar{A}-I_{n}=\left[\begin{array}{cc}
-0.5 & 0.1 \\
0.2 & -0.6
\end{array}\right] \text {. }
$$

Using (3.5) for $n=2$ we obtain

$$
\hat{A}_{1}^{(1)}=\hat{a}_{11}-\frac{\hat{a}_{12} \hat{a}_{21}}{\hat{a}_{22}}=-0.5+\frac{0.1 * 0.2}{0.6}<1 .
$$

Condition i) of Theorem 3.5 is satisfied and the positive system (3.1) with (3.7) is asymptotically stable.

Similarly, using the elementary row operations to the matrix (3.8) we obtain

$$
\hat{A}=\left[\begin{array}{cc}
-0.5 & 0.1 \\
0.2 & -0.6
\end{array}\right] \stackrel{{ }^{[}\left[1+2 \times \frac{1}{6}\right]}{\longrightarrow}\left[\begin{array}{cc}
-\frac{7}{15} & 0 \\
0.2 & -0.6
\end{array}\right] \text {. }
$$

The condition ii) of Theorem 3.5 is satisfied and the positive system is asymptotically stable.

\section{LINEAR SYSTEMS WITH DELAYS}

Consider the continuous-time linear system with $q$ delays (Kaczorek 2009c)

$$
\dot{x}(t)=A_{0} x(t)+\sum_{k=1}^{q} A_{k} x\left(t-d_{k}\right)
$$

where $x(t) \in \mathfrak{R}^{n}$ is the state vector, $A_{k} \in \mathfrak{R}^{n \times n}$, $k=0,1, \ldots, q$ and $d_{k}>0, \quad k=1, \ldots, q$ are delays.

The initial conditions for (4.1) have the form

$$
x(t)=x_{0}(t) \text { for } t \in[-d, 0], d=\max _{k} d_{k}
$$

The system (4.1) is called (internally) positive if $x(t) \in \Re_{+}^{n}, t \geq 0$ for any initial conditions $x_{0}(t) \in \Re_{+}^{n}$.

Theorem 4.1. The system (4.1) is positive if and only if

$$
A_{0} \in M_{n} \text { and } A_{k} \in \Re_{+}^{n \times n}, k=1, \ldots, q
$$

where $M_{n}$ is the set of $n \times n$ Metzler matrices.

Proof is given in (Kaczorek 2009c).

Theorem 4.2. The positive system with delays (4.1) is asymptotically stable if and only if the positive system without delays

$$
\dot{x}=A x, A=\sum_{k=0}^{q} A_{k} \in M_{n}
$$

is asymptotically stable.

Proof is given in (Kaczorek 2009c).

To check the asymptotic stability of the system (4.1) Theorem 2.5 is recommended. The application of Theorem 2.5 to checking the asymptotic stability of the system (4.1) will be illustrated by the following example.

Example 4.1. Consider the system (4.1) with $q=1$ and the matrices 


$$
A_{0}=\left[\begin{array}{cc}
-1 & 0.2 \\
0.2 & -1.4
\end{array}\right], \quad A_{1}=\left[\begin{array}{cc}
0.5 & 0.1 \\
0.2 & 0.8
\end{array}\right]
$$

The matrix of the positive system (4.4) with delays has the form

$$
A=A_{0}+A_{1}=\left[\begin{array}{cc}
-0.5 & 0.3 \\
0.4 & -0.6
\end{array}\right] \in M_{2} .
$$

Using (2.6) for the matrix (4.6) we obtain

$$
\hat{A}_{1}^{(1)}=-0.5+\frac{0.4 * 0.3}{0.6}=-0.3 .
$$

Condition i) of Theorem 2.5 is satisfied and the positive system (4.1) with (4.5) is asymptotically stable.

Now let us consider the discrete-time linear system with $q$ delays (Busłowicz 2008)

$$
x_{i+1}=\sum_{k=0}^{q} A_{k} x_{i-k}, \quad i \in Z_{+}
$$

where $x_{i} \in \mathfrak{R}^{n}$ is the state vector and $A_{k} \in \mathfrak{R}^{n \times n}$, $k=0,1, \ldots, q$.

The initial conditions for (4.8) have the form

$$
x_{-k} \in \Re^{n} \text { for } k=0,1, \ldots, q .
$$

The system (4.8) is called (internally) positive if $x_{i} \in \mathfrak{R}_{+}^{n}, i \in Z_{+}$for any initial conditions $x_{-k} \in \mathfrak{R}_{+}^{n}$ for $k=0,1, \ldots, q$.

Theorem 4.3. (Kaczorek 2002) The system (4.8) is positive if and only if $A_{k} \in \mathfrak{R}_{+}^{n \times n}, k=0,1, \ldots, q$.

Theorem 4.4. The positive discrete-time system with delays (4.8) is asymptotically stable if and only if the positive system without delays

$$
x_{i+1}=\bar{A} x_{i}, \bar{A}=\sum_{k=0}^{q} A_{k}, i \in Z_{+}
$$

is asymptotically stable.

Proof is given in (Busłowicz 2008).

To check the asymptotic stability of the system (4.8) Theorem 3.5 is recommended. The application of Theorem 3.5 to checking the asymptotic stability of the system (4.8) will be illustrated by the following example.

Example 4.2. Consider the positive system (4.8) with $q=1$ and the matrices

$$
A_{0}=\left[\begin{array}{ll}
0.2 & 0.2 \\
0.1 & 0.2
\end{array}\right], \quad A_{1}=\left[\begin{array}{ll}
0.2 & 0.1 \\
0.1 & 0.3
\end{array}\right] .
$$

The matrix of the positive system (4.10) without delays has the form

$$
\bar{A}=A_{0}+A_{1}=\left[\begin{array}{ll}
0.4 & 0.3 \\
0.2 & 0.5
\end{array}\right] \text {. }
$$

In this case

$$
\hat{A}=\bar{A}-I_{n}=\left[\begin{array}{cc}
-0.6 & 0.3 \\
0.2 & -0.5
\end{array}\right]
$$

and using the elementary row operation to (4.13) we obtain

$$
\left[\begin{array}{cc}
-0.6 & 0.3 \\
0.2 & -0.5
\end{array}\right] \stackrel{{ }^{\left[1+2 \times \frac{3}{5}\right.}}{\longrightarrow}\left[\begin{array}{cc}
-0.48 & 0 \\
0.2 & -0.5
\end{array}\right]
$$

The condition ii) of Theorem 3.5 is satisfied and the positive system is asymptotically stable

\section{D LINEAR SYSTEMS}

Consider the general autonomous model of 2D linear systems

$$
x_{i+1, j+1}=A_{0} x_{i, j}+A_{1} x_{i+1, j}+A_{2} x_{i, j+1}, \quad i, j \in Z_{+}
$$

where $x_{i, j} \in \mathfrak{R}^{n}$ is the state vector and $A_{k} \in \mathfrak{R}^{n \times n}$, $k=0,1,2$.

Boundary conditions for (5.1) have the form

$$
x_{i, 0} \in \mathfrak{R}^{n}, i \in Z_{+} \text {and } x_{0, j} \in \mathfrak{R}^{n}, j \in Z_{+} .
$$

The model (5.1) is called (internally) positive if $x_{i, j} \in \mathfrak{R}_{+}^{n}, i, j \in Z_{+}$for any initial conditions $x_{i, 0} \in \mathfrak{R}_{+}^{n}$, $i \in Z_{+}, x_{0, j} \in \Re_{+}^{n}, j \in Z_{+}$.

Theorem 5.1. (Kaczorek 2002) The system (5.1) is positive if and only if

$$
A_{k} \in \mathfrak{R}_{+}^{n \times n}, k=0,1,2 .
$$

The Roesser autonomous model of 2D linear systems has the form (Kaczorek 2002)

$$
\left[\begin{array}{c}
x_{i+1, j}^{h} \\
x_{i, j+1}^{v}
\end{array}\right]=\left[\begin{array}{ll}
A_{11} & A_{12} \\
A_{21} & A_{22}
\end{array}\right]\left[\begin{array}{c}
x_{i, j}^{h} \\
x_{i, j}^{v}
\end{array}\right], \quad i, j \in Z_{+}
$$

where $x_{i, j}^{h} \in \mathfrak{R}^{n_{1}}$ and $x_{i, j}^{v} \in \mathfrak{R}^{n_{2}}$ are the horizontal and vertical state vectors at the point $(i, j)$ and $A_{k, l} \in \mathfrak{R}^{n_{k} \times n_{l}}$, $k, l=1,2$.

Boundary conditions for (5.4) have the form 


$$
x_{0, j}^{h} \in \mathfrak{R}^{n_{1}}, j \in Z_{+} \text {and } x_{i, 0}^{v} \in \mathfrak{R}^{n_{2}}, i \in Z_{+} .
$$

The model (5.4) is called (internally) positive if $x_{i, j}^{h} \in \mathfrak{R}_{+}^{n_{1}}$ and $x_{i, j}^{v} \in \mathfrak{R}_{+}^{n_{2}}$ for any initial conditions $x_{0, j}^{h} \in \mathfrak{R}_{+}^{n_{1}}, j \in Z_{+}$and $x_{i, 0}^{v} \in \mathfrak{R}_{+}^{n_{2}}, i \in Z_{+}$.

Theorem 5.2. (Kaczorek 2002) The Roesser model (5.4) is positive if and only if

$$
\left[\begin{array}{ll}
A_{11} & A_{12} \\
A_{21} & A_{22}
\end{array}\right] \in \mathfrak{R}_{+}^{n \times n}, n=n_{1}+n_{2} .
$$

The positive general model (5.1) is called asymptotically stable if

$$
\lim _{i, j \rightarrow \infty} x_{i, j}=0 \text { for all } x_{i, 0} \in \mathfrak{R}_{+}^{n}, i \in Z_{+}, x_{0, j} \in \mathfrak{R}_{+}^{n}, j \in Z_{+} .
$$

Similarly, the positive Roesser model (5.4) is called asymptotically stable if

$$
\begin{gathered}
\lim _{i, j \rightarrow \infty}\left[\begin{array}{c}
x_{i, j}^{h} \\
x_{i, j}^{v}
\end{array}\right]=0 \text { for all } x_{0, j}^{h} \in \Re_{+}^{n_{1}}, j \in Z_{+}, \\
x_{i, 0}^{v} \in \Re_{+}^{n_{2}}, i \in Z_{+} .
\end{gathered}
$$

Theorem 5.3. The positive general model (5.1) is asymptotically stable if and only if the positive 1D system

$$
x_{i+1}=A x_{i}, \quad A=A_{0}+A_{1}+A_{2}, \quad i \in Z_{+}
$$

is asymptotically stable.

Proof is given in (Kaczorek 2009a and 2009d).

Theorem 5.4. The positive Roesser model (5.4) is asymptotically stable if and only if the positive 1D system

$$
x_{i+1}=\left[\begin{array}{ll}
A_{11} & A_{12} \\
A_{21} & A_{22}
\end{array}\right] x_{i}, \quad i \in Z_{+}
$$

is asymptotically stable.

Proof is given in (Kaczorek 2009a and 2009d).

To check the asymptotic stability of the positive general model (5.1) and the positive Roesser model (5.4) the Theorem 3.5 is recommended. The application of Theorem 3.5 to checking the asymptotic stability of the models (5.1) and (5.4) will be shown on the following examples.

Example 5.1. Consider the positive general model (5.1) with the matrix

$$
A_{0}=\left[\begin{array}{ll}
0.1 & 0.2 \\
0.1 & 0.1
\end{array}\right], \quad A_{1}=\left[\begin{array}{ll}
0 & 0.1 \\
0 & 0.1
\end{array}\right], \quad A_{1}=\left[\begin{array}{ll}
0.2 & 0.3 \\
0.1 & 0.2
\end{array}\right]
$$

$$
A=A_{0}+A_{1}+A_{2}=\left[\begin{array}{ll}
0.3 & 0.6 \\
0.2 & 0.4
\end{array}\right]
$$

and

$$
\hat{A}=\bar{A}-I_{n}=\left[\begin{array}{cc}
-0.7 & 0.6 \\
0.2 & -0.6
\end{array}\right]
$$

Using the elementary row operation to (5.13) we obtain

$$
\hat{A}=\left[\begin{array}{cc}
-0.7 & 0.6 \\
0.2 & -0.6
\end{array}\right] \stackrel{\left.{ }_{L[1+2 \times 1}\right]}{\longrightarrow}\left[\begin{array}{cc}
-0.5 & 0 \\
0.2 & -0.6
\end{array}\right] \text {. }
$$

The condition ii) of Theorem 3.5 is satisfied and the positive general model with (5.11) is asymptotically stable.

Example 5.2. Consider the positive Roesser model (5.4) with the matrices

$$
A=\left[\begin{array}{ll}
A_{11} & A_{12} \\
A_{21} & A_{22}
\end{array}\right]
$$

And

$$
\begin{aligned}
& A_{11}=\left[\begin{array}{ll}
0.3 & 0.2 \\
0.1 & 0.4
\end{array}\right], \quad A_{12}=\left[\begin{array}{l}
0.1 \\
0.2
\end{array}\right], \\
& A_{21}=\left[\begin{array}{ll}
0.2 & 0.1],
\end{array} A_{22}=[0.8] .\right.
\end{aligned}
$$

In this case

$$
\hat{A}=A-I_{n}=\left[\begin{array}{ccc}
-0.7 & 0.2 & 0.1 \\
0.1 & -0.6 & 0.2 \\
0.2 & 0.1 & -0.2
\end{array}\right]
$$

Using the elementary row operation to (5.15) we obtain

$$
\begin{aligned}
& {\left[\begin{array}{ccc}
-0.7 & 0.2 & 0.1 \\
0.1 & -0.6 & 0.2 \\
0.2 & 0.1 & -0.2
\end{array}\right] \stackrel{\underset{L}{L[2+3 \times 3 \times 0.5]} \underset{0.3}{L}}{\longrightarrow}\left[\begin{array}{ccc}
-0.6 & 0.25 & 0 \\
0.3 & -0.5 & 0 \\
0.2 & 0.1 & -0.2
\end{array}\right]} \\
& \stackrel{\text { L1+2×0.5] }}{\longrightarrow}\left[\begin{array}{ccc}
-0.45 & 0 & 0 \\
0.3 & -0.5 & 0 \\
0.2 & 0.1 & -0.2
\end{array}\right]
\end{aligned}
$$

The condition ii) of Theorem 3.5 is satisfied and the positive Roesser model with (5.14) is asymptotically stable.

In a similar way as for 1D linear systems using (Kaczorek 2009b) the considerations can be easily extended to 2D linear systems with delays and to fractional 1D and 2D linear systems.

In this case 


\section{CONCLUDING REMARKS}

New tests for checking asymptotic stability of positive 1D continuous-time and discrete-time linear systems without and with delays and of positive 2D linear systems described by the general and the Roesser models have been proposed. The tests are based on the Theorem 2.5 and Theorem 3.5. Checking of the asymptotic stability of positive 2D linear systems has been reduced to checking of suitable corresponding 1D positive linear systems. The tests can be also extended to $2 \mathrm{D}$ continuous-discrete linear systems and to $1 \mathrm{D}$ and 2D fractional linear systems. An open problem is extension of these considerations to $2 \mathrm{D}$ positive switched linear systems.

\section{ACKNOWLEDGMENT}

This work was supported by Ministry of Science and Higher Education in Poland under work S/WE/1/2011.

\section{REFERENCES}

Busłowicz M. 2008. "Simple stability conditions for linear positive discrete-time systems with delays." Bull. Pol. Acad. Sci. Techn., vol. 56, no. 4, 325-328.

Busłowicz M. 2010. "Robust stability of positive discrete-time linear systems of fractional order." Bull. Pol. Acad. Sci. Techn., vol. 58, no. 4, 567-572.

Farina L. and S. Rinaldi 2000. Positive Linear Systems; Theory and Applications, J. Wiley, New York.

Kaczorek T. 2002. Positive $1 D$ and $2 D$ Systems, Springer Verlag, London.

Kaczorek T. 2009a. "Asymptotic stability of positive 2D linear systems." Computer Applications in Electrical Engineering, Poznan University of Technology, Institute of Electrical Engineering and Electronics, Electrical Engineering Committee of Polish Academy of Sciences, IEEE Poland Section.

Kaczorek T. 2009b. "Asymptotic stability of positive 2D linear systems with delays." Bull. Pol. Acad. Sci. Techn., vol. 57, no. 2, 133-138.

Kaczorek T. 2009c. "Stability of positive continuous-time linear systems with delays." Bull. Pol. Acad. Sci. Techn., vol. 57, no. 4, 395-398.

Kaczorek T. 2009d. "Independence of asymptotic stability of positive 2D linear systems with delays of their delays." Int. J. Appl. Math. Comput. Sci., vol. 19, no. 2, 255-261.

Kaczorek T. 2010. "Stability and stabilization of positive fractional linear systems by state-feedbacks." Bull. Pol. Acad. Sci. Techn., vol. 58, no. 4, 517-554.

Narendra K.S. and R. Shorten. 2010. "Hurwitz Stability of Metzler Matrices.” IEEE Trans. Autom. Contr., Vol. 55, no. 6 June $1484-1487$.

\section{AUTHOR BIOGRAPHIES}

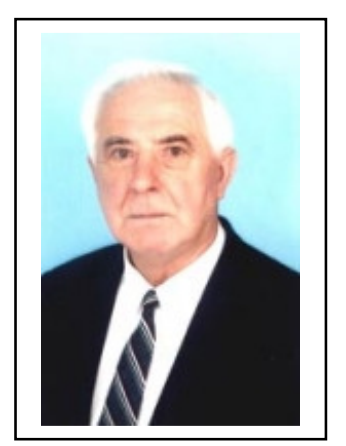

TADEUSZ KACZOREK born 27.04.1932 in Poland, received the $\mathrm{MSc}$., $\mathrm{PhD}$ and DSc degrees from Electrical Engineering of Warsaw University of Technology in 1956, 1962 and 1964, respectively. In the period 1968 - 69 he was the dean of Electrical Engineering Faculty and in the period $1970-73$ he was a deputy rector of Warsaw University of Technology. Since 1971 he has been professor and since 1974 full professor at Warsaw University of Technology. In 1986 he was elected a corresponding member and in 1996 full member of Polish Academy of Sciences. In the period 1988 - 1991 he was the director of the Research Centre of Polish Academy of Sciences in Rome. In June 1999 he was elected the full member of the Academy of Engineering in Poland. In May 2004 he was elected the honorary member of the Hungarian Academy of Sciences. He was awarded by the University of Zielona Gora (2002) by the title doctor honoris causa, the Technical University of Lublin (2004), the Technical University of Szczecin (2004), Warsaw University of Technology (2004), Bialystok Technical University (2008), Lodz Technical University (2009) and Opole Technical University (2009).

His research interests cover the theory of systems and the automatic control systems theory, specially, singular multidimensional systems, positive multidimensional systems and singular positive $1 \mathrm{D}$ and $2 \mathrm{D}$ systems. He has initiated the research in the field of singular $2 \mathrm{D}$, positive 2D linear systems and positive fractional 1D and 2D systems. He has published 24 books (7 in English) and over 950 scientific papers.

He supervised $69 \mathrm{Ph} . \mathrm{D}$. theses. More than 20 of this $\mathrm{PhD}$ students became professors in USA, UK and Japan. $\mathrm{He}$ is editor-in-chief of the Bulletin of the Polish Academy of Sciences: Technical Sciences and editorial member of about ten international journals. His e-mail address is : kaczorek@isep.pw.edu.pl. 